PAPER

\title{
Ultrasonic direction measurement using sensitivity-compensated signal and band expansion method based on linear prediction
}

\author{
Dai Chimura*, Ryo Toh and Seiichi Motooka \\ Chiba Institute of Technology, \\ 2-17-1 Tsudanuma, Narashino, 275-0016 Japan \\ (Received 4 February 2016, Accepted for publication 13 September 2016)
}

\begin{abstract}
High-resolution ultrasonic pulse-echo measurement is discussed. For higher time resolution, a sensitivity-compensated (SC) signal and a method of band expansion using linear prediction (LP) processing have been proposed. Moreover, two-dimensional direction measurement calculated from the difference in distances derived using the SC signal has been studied. Because a small error in distances will result in a large error in the direction result, higher accuracy of distance measurement is required. In this paper, the efficiency of band expansion using LP processing with an autoregressive model is discussed for two-dimensional direction measurement. The comparison results show that by using the band expansion method, the compressed pulse width is shortened to about $18 \%$ of its original value and the accuracy of direction measurement is improved using the higher-timeresolution signal.
\end{abstract}

Keywords: Pulse compression, Sensitivity compensated signal, Target ranging, Linear prediction

PACS number: 43.58.+z [doi:10.1250/ast.38.71]

\section{INTRODUCTION}

Ultrasonic sensing is used in various media such as air, water and soil [1-4]. An ultrasonic pulse-echo method using the time of flight (TOF) is often used for target detection in air such as by robots and automobiles [5-7]. For high-accuracy TOF determination, a higher signal-tonoise ratio (SNR) and higher time resolution are needed. Thus, a pulse compression technique is employed for TOF measurements. For pulse compression with a higher SNR and higher resolution, a signal with a long pluse width and wide bandwidth, such as a linear-frequency-modulated signal (chirp wave) is employed as a transmitting signal. Moreover, a coded signal such as a maximum-length sequence signal has been studied for multiple-transmittance measurement $[8,9]$.

However, the efficiency of pulse compression is limited owing to the transfer function containing the sensitivities of ultrasonic transducers. In our previous works, for pulse compression with higher resolution, an amplitude-modulated chirp wave that compensates sensitivities was proposed as a sensitivity-compensated (SC) signal and TOF measurements were studied [10-17]. The SC signal is calculated from a chirp wave and by inverse filtering of the measured signal, which is mainly affected by the sensitiv-

*e-mail: chimura.d@gmail.com ities of ultrasonic transducers. The spectrum of the transmitting signal is also compensated to obtain a received signal with a broader and flatter spectrum. In particular, the sensitivity of a conventional aerial transducer has a property that the $-3 \mathrm{~dB}$ bandwidth is narrow while the bandwidth above $-30 \mathrm{~dB}$ is rather wide. Therefore, these transducers have the potential to be compensated to achieve higher-resolution measurement.

Here, considering a received signal with a flatter spectrum, a band expansion method using linear prediction (LP) processing [18,19] has been proposed for TOF measurement with much higher time resolution. The predicted value derived from the components of the flatter spectrum with a higher SNR compensates the spectrum with a lower SNR, and it is expected that a signal with higher time resolution can be obtained. We have studied one-dimensional target ranging using an SC signal and LP processing with an autoregressive (AR) model [20,21]. The results showed that, using the method of band expansion with the AR model, the time resolution of the compressed pulse was improved.

On the other hand, two-dimensional direction measurement using an SC signal has been studied [22,23]. For direction measurement, two receivers are employed and two propagation distances are obtained for direction calculation. Here, even if the distance errors are small, the relative error in the direction can be large. Therefore, 
higher-accuracy target ranging is needed for direction measurement.

Also, the affect of directivity on SC processing or the LP band expansion method has not been discussed sufficiently. Because the directivity is frequency-dependent, the efficiency of the band expansion method will be affected by the direction of reflecting objects.

In this paper, two-dimensional direction measurement using an SC signal with LP processing is discussed. Moreover, the affect of directivity on the band expansion method is studied by simulation and experimentally.

Accordingly, the time resolution is improved by the band expansion method, and the accuracy of direction measurement is improved when the spectrum of the compressed pulse is comparatively flat.

\section{THEORY}

\subsection{Theory of Sensitivity-Compensated Signal}

Neglecting noise, the spectrum of a receiving signal $F_{\mathrm{R}}(\omega)$ can be expressed as $F_{\mathrm{T}}(\omega) \cdot R(\omega)$, where $F_{\mathrm{T}}(\omega)$ and $R(\omega)$ are, respectively, the spectrum of the transmitting signal and the transfer function, which contains the sensitivities of the transmitter and receiver. Here, if we use a transmitting signal with the amplitude characteristic of $|R(\omega)|^{-1}$, a signal with a flat spectrum can be received. Theoretically, the ideal amplitude characteristic of the spectrum of a transmitting signal can be calculated by

$$
\frac{\left|F_{\mathrm{T}}(\omega)\right|}{\left|F_{\mathrm{R}}(\omega)\right|}=\frac{1}{|R(\omega)|} .
$$

In our study, the spectrum of the SC signal $F_{C}(\omega)$, calculated as the inverse-filtered received signal, is given as

$$
F_{\mathrm{C}}(\omega)=\frac{\left|F_{\mathrm{R}}(\omega)\right|}{\left|F_{\mathrm{R}}(\omega)\right|^{2}+\alpha^{2} \cdot\left|F_{\mathrm{R}}(\omega)_{\max }\right|^{2}} \cdot F_{\mathrm{T}}(\omega),
$$

where $\alpha$ is a stabilization factor limiting the divergence of the response function when the value of $F_{\mathrm{R}}(\omega)$ is small.

Here, if we use a linear FM chirp wave as $F_{\mathrm{T}}(\omega)$, the inverse-filtered amplitude characteristics will be linearly reflected in the time domain waveform and an amplitudemodulated chirp wave will be derived. By using the SC signal as a transmitting signal, a signal with a broader and flatter spectrum can be received.

\subsection{Theory of Band Expansion by Linear Prediction}

Considering that a signal with a flatter spectrum can be received by transmitting the SC signal, a method of band expansion using LP processing is proposed for TOF determination with much higher time resolution. For band expansion, predicted values are calculated from LP coefficients derived from the flatter spectrum with a higher SNR. Because the predicted values compensate the spectrum with a lower SNR, it is expected that a signal with higher time resolution can be obtained. In this paper, an AR model is employed for LP processing.

In the AR model, the spectrum of signal $F(\omega)$ is calculated from a linear combination of the previous values and AR coefficients $a_{i}$ as follows:

$$
F(\omega)=-\sum_{i=1}^{p} a_{i} \cdot F(\omega-i \cdot \Delta \omega)+e(\omega),
$$

where $p$ and $e(\omega)$ are the $\mathrm{AR}$ order and prediction error, respectively, and $\Delta \omega$ is the discrete interval of $\omega$.

Here, $a_{i}$ can be derived from the least mean square of $e(\omega)$. The normal equation of $a_{i}$ is

$$
\varphi(i)+\sum_{k=1}^{p} a_{k} \cdot \varphi(k-i)=0(i=1,2, \cdots, p),
$$

where $\varphi(i)$ is the autocorrelation of the spectrum $F(\omega)$ at $i \cdot \Delta \omega$.

Equation (4) can be expressed as

$$
\begin{aligned}
& {\left[\begin{array}{cccc}
\varphi(0) & \varphi(1) & \cdots & \varphi(p-1) \\
\varphi(1) & \varphi(0) & \cdots & \varphi(p-2) \\
\vdots & \vdots & & \vdots \\
\varphi(p-1) & \varphi(p-2) & \cdots & \varphi(0)
\end{array}\right]\left[\begin{array}{c}
a_{1} \\
a_{2} \\
\vdots \\
a_{p}
\end{array}\right]} \\
& =-\left[\begin{array}{c}
\varphi(1) \\
\varphi(2) \\
\vdots \\
\varphi(p)
\end{array}\right] .
\end{aligned}
$$

By using $a_{i}$, the spectrum with the lower SNR is compensated by the predicted value $F_{\mathrm{A}}(\omega)$, given as

$$
F_{\mathrm{A}}(\omega)=-\sum_{i=1}^{p} a_{i} \cdot F_{\mathrm{A}}(\omega-i \cdot \Delta \omega) .
$$

Here, for previous values $F_{\mathrm{A}}(\omega-i \cdot \Delta \omega)$, the true value is employed for the spectrum with the higher SNR, while the predicted value is employed for the spectrum with the lower SNR.

For band expansion, the real and imaginary parts of the spectrum are calculated separately.

\subsection{Theory of Pulse Compression}

The TOF is determined by the pulse peak of the compressed signal waveform. The cross-correlation of the spectrum of the received signal $F_{\mathrm{R}}(\omega)$ and the spectrum of a reference signal $F_{0}(\omega)$ is employed for pulse compression as follows:

$$
F_{\mathrm{P}}(\omega)=F_{\mathrm{R}}(\omega) \cdot F_{0}^{*}(\omega),
$$

where $F_{\mathrm{p}}(\omega)$ and $F_{0}^{*}(\omega)$ are the spectrum of the pulse compressed signal and the spectrum of the complex conjugate of $F_{0}(\omega)$, respectively. 


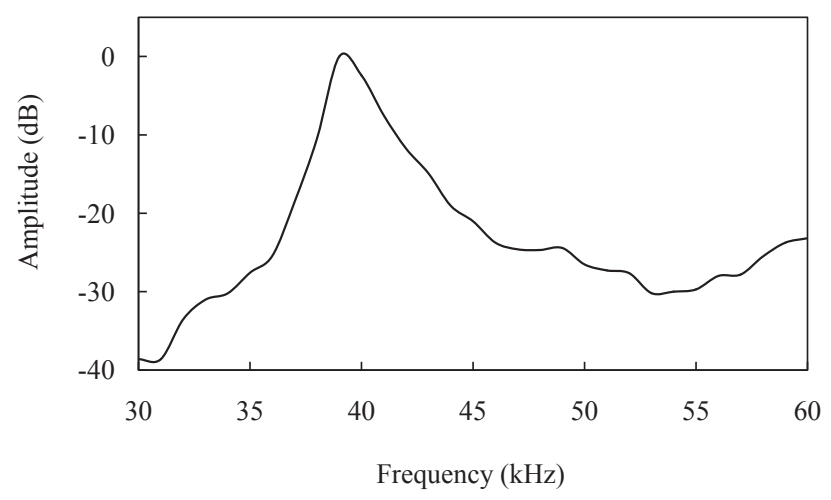

Fig. 1 Sensitivity of transducers.

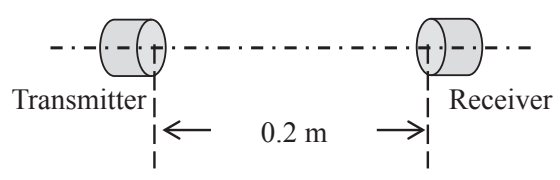

Fig. 2 Arrangement used for SC signal derivation.

\section{DERIVATION OF SENSITIVITY- COMPENSATED SIGNAL}

The sensitivities of conventional aerial transducers have a property that the $-3 \mathrm{~dB}$ bandwidth is narrow while the bandwidth above $-30 \mathrm{~dB}$ is rather wide. Therefore, the spectrum of the received signal has a bandwidth that can potentially be compensated. Figure 1 shows the sensitivity of conventional aerial transducers with a $40 \mathrm{kHz}$ resonant peak. In this figure, under $-3 \mathrm{~dB}$, the sensitivity characteristic can potentially be compensated to receive a signal with a broader and flatter spectrum. To derive the SC signal, a transmitter (Murata Manufacturing Co.: MA40A4S) and receiver (G.R.A.S.: TYPE-4158N) are positioned with the direct transmitting-receiving arrangement shown in Fig. 2. By using a chirp wave as a transmitting signal, the received signal, which is mainly affected by the sensitivities of the transducers, is measured. In this paper, as the chirp wave, a linear FM pulse signal with a pulse width of $5 \mathrm{~ms}$ is employed, and the frequency is modulated from 35 to $55 \mathrm{kHz}$. Here, the received signal using the chirp wave is abbreviated because the spectrum is similar to that in Fig. 1. Then, the SC signal is calculated from the chirp wave and the received signal as Eq. (2), where $\alpha=0.03$ is employed to compensate the spectrum of the received signal considering the sensitivities above about $-26 \mathrm{~dB}$ shown in Fig. 1. For pulse compression, the reference signal using the SC signal as the transmitting signal is measured with the arrangement shown in Fig. 2. Here, the average of 50 received signals is employed. Figure 3 shows the reference signal using the SC signal. As shown, the reference signal has a broader and flatter

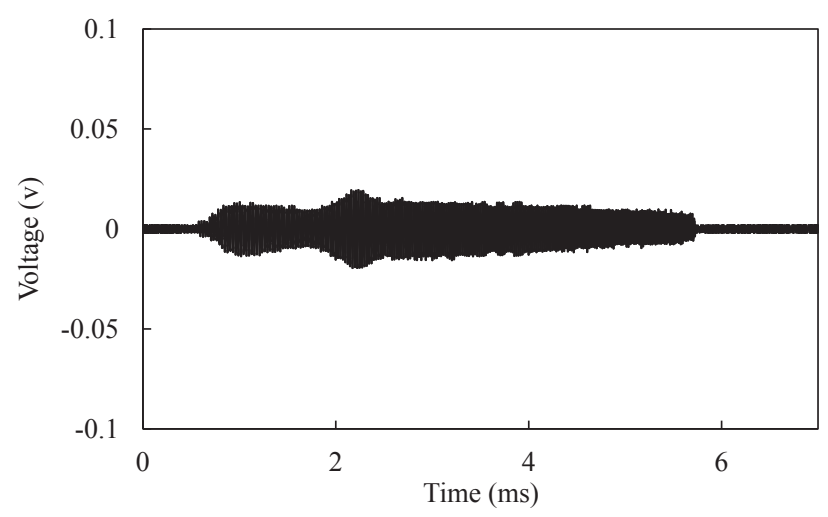

(a)

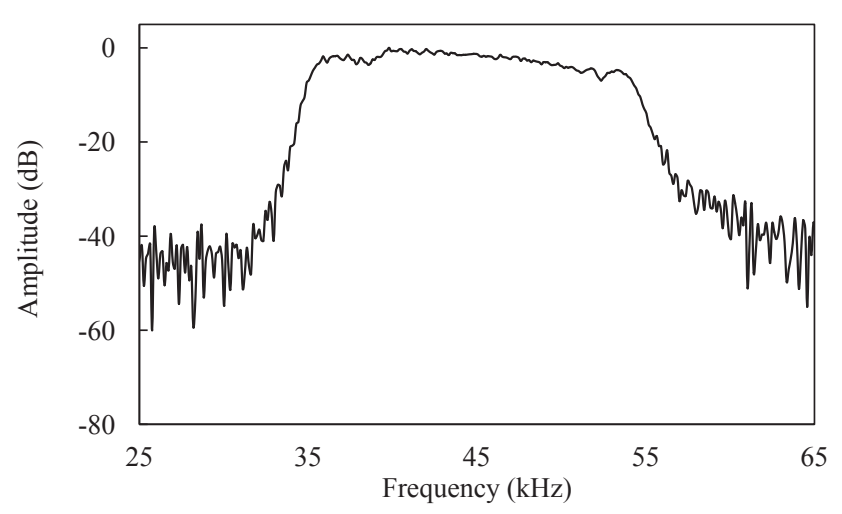

(b)

Fig. 3 Reference signal using the SC signal: (a) waveform and (b) spectrum.

spectrum compared with the characteristic of the sensitivity of the transducers shown in Fig. 1.

\section{SIMULATION OF BAND EXPANSION}

\subsection{Time Resolution of Band-Expanded Signal}

By using the LP band expansion processing with a flatter spectrum, a signal with higher time resolution will be obtained. However, the relationship between the efficiency of band expansion and the evenness of the spectrum should be discussed.

In this section, the efficiency of the band expansion method considering the evenness of the spectrum is discussed. In this paper, to derive the SC signal, transducers are positioned with a direct transmitting-receiving arrangement. Then, in the direction measurement, the received signal is also affected by the directivities of the transducers. Because the directivity is frequency-dependent, it can be considered that the efficiencies of the SC signal and the LP band expansion will be affected by the direction of reflecting objects. In this simulation, the discussion is limited to the directivity, and other factors such as the sensitivities of ultrasonic transducers and noise are neglected.

Figure 4 shows the arrangement of the band expansion simulation. A transmitter (radius: $5 \mathrm{~mm}$ ) and a receiver 


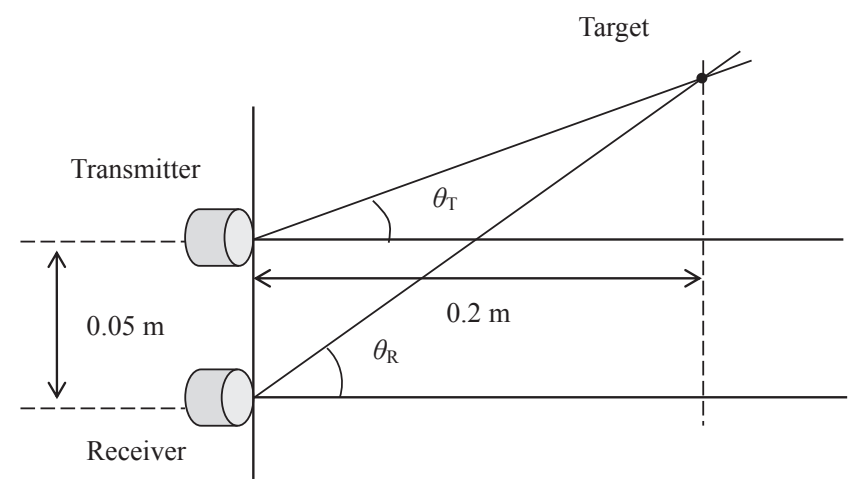

Fig. 4 Arrangement used for simulation of band expansion.

(radius: $3 \mathrm{~mm}$ ) are arranged in parallel. The target is arranged with an angle $\theta_{\mathrm{T}}$ formed by the transmitter and an angle $\theta_{\mathrm{R}}$ formed by the receiver. The effect of the directivity is calculated using the directivity function $D(\theta, \omega)$ given as

$$
\begin{aligned}
& D(\theta, \omega)=\frac{2 J_{1}(z)}{z}, \\
& z=\frac{2 \pi}{\lambda} r \sin \theta=\frac{\omega}{c} r \sin \theta,
\end{aligned}
$$

where $J_{1}(z)$ is the Bessel function of $z$ of the first kind of order 1. Here, $r, \lambda$ and $c$ are the radius of the ultrasonic transducers, the wavelength and the sound velocity, respectively.

The spectrum of the estimated received signal $F_{\mathrm{ER}}(\omega)$ is calculated from the directivity function of the transmitter $D_{\mathrm{T}}\left(\theta_{\mathrm{T}}\right)$ and that of the receiver $D_{\mathrm{R}}\left(\theta_{\mathrm{R}}\right)$ as follows:

$$
F_{\mathrm{ER}}(\omega)=F_{\mathrm{CW}}(\omega) \cdot D_{\mathrm{T}}\left(\theta_{\mathrm{T}}, \omega\right) \cdot D_{\mathrm{R}}\left(\theta_{\mathrm{R}}, \omega\right),
$$

where $F_{\mathrm{CW}}(\omega)$ is the transmitting signal. Here, the reflectivity of the target is assumed to be 1 . The waveform of the estimated received signal is derived by the inverse Fourier transform of the spectrum. As the transmitting signal, an FM pulse signal (chirp wave) with a pulse width of $5 \mathrm{~ms}$ and a modulated frequency of $35-55 \mathrm{kHz}$ is employed. Then, pulse compression is performed by crosscorrelation of the chirp wave and the estimated received signal.

Figure 5 shows the compressed signal for the arrangement with $\theta_{\mathrm{T}}=0^{\circ}$. The pulse width of the envelope curve at $-3 \mathrm{~dB}$ is $44.6 \mu \mathrm{s}$ and the spectrum is nearly flat. Then, the band expansion method is applied to the compressed signal, and the band-expanded signal obtained by simulation is shown in Fig. 6. Because the predicted values compensate the spectrum where the bandwidth has a lower SNR, the waveform of the band-expanded signal becomes similar to that of the impulse signal. Here, $p=26$ is employed for the LP calculation.

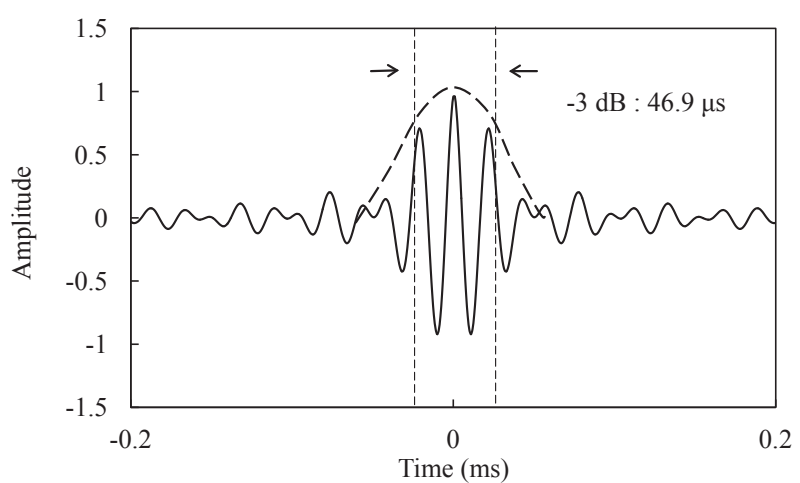

(a)

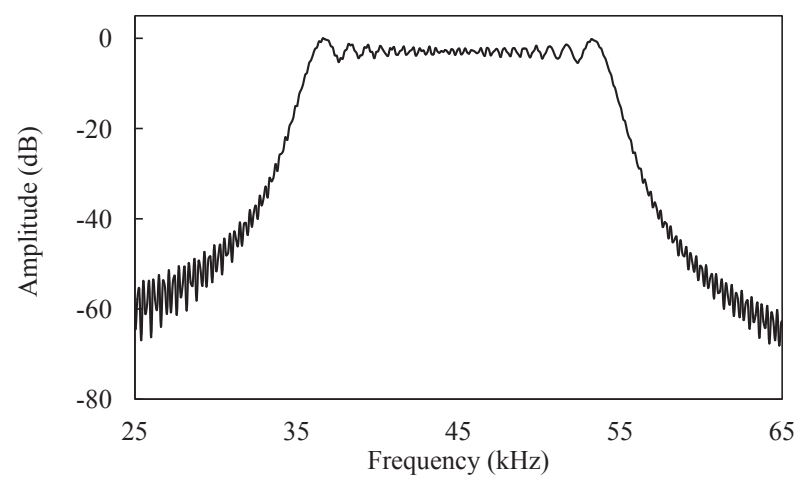

(b)

Fig. 5 Compressed signal for the arrangement with $\theta_{\mathrm{T}}=0^{\circ}$ : (a) waveform and (b) spectrum.

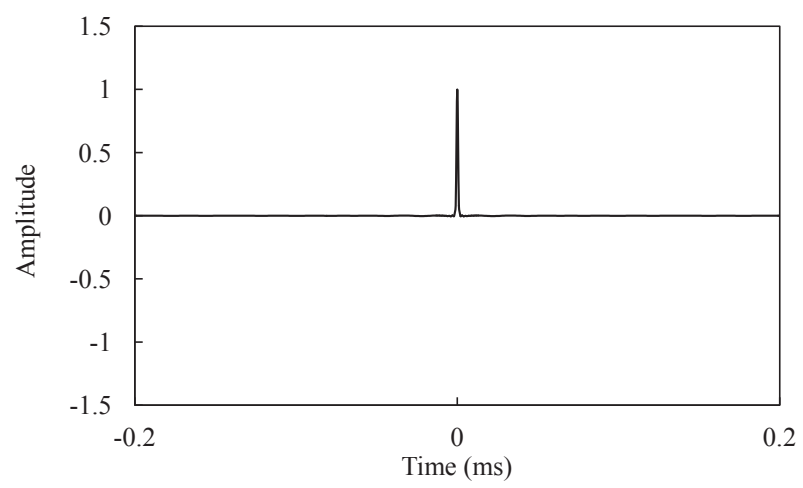

(a)

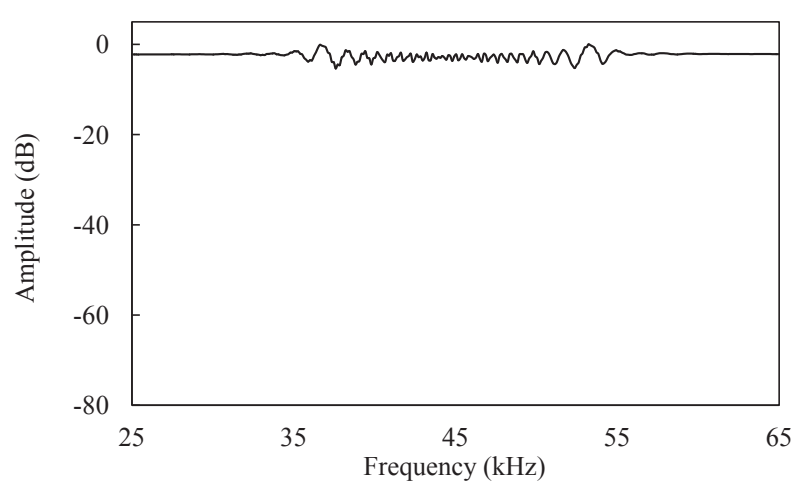

(b)

Fig. 6 Band-expanded signal derived from compressed signal $\left(\theta_{\mathrm{T}}=0^{\circ}\right)$ : (a) waveform and (b) spectrum. 


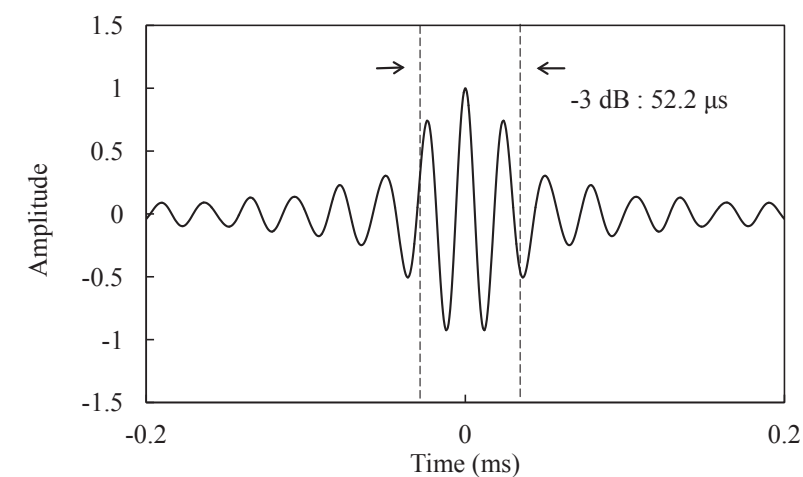

(a)

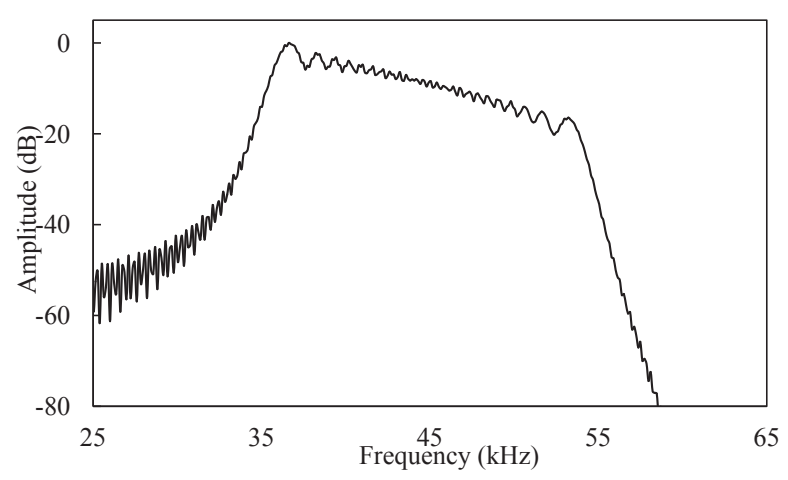

(b)

Fig. 7 Compressed signal for the arrangement with $\theta_{\mathrm{T}}=45^{\circ}$ : (a) waveform and (b) spectrum.

Figures 7 and 8 show the compressed signal of the arrangement with $\theta_{\mathrm{T}}=45^{\circ}$ and the band-expanded signal, respectively. The spectrum of the compressed signal becomes uneven because the higher-frequency component is more affected by the directivity. As shown in Figs. 7 and 8 , the $-3 \mathrm{~dB}$ pulse widths are $52.2 \mu \mathrm{s}$ and $20.1 \mu \mathrm{s}$, respectively. By using the band expansion, the pulse width is shortened to about $39 \%$. However, the efficiency of the band expansion method is clearly reduced. By comparing Fig. 5 and Fig. 7, it can be concluded that the directivity will also affect the efficiency of the SC processing method.

In this simulation, the time resolution of the signal was improved by using the band expansion method. On the other hand, when the signal was affected by the directivity, that is, the spectrum of the signal became uneven, the efficiency of the band expansion was decreased.

\subsection{Phase Spectrum of Band-Expanded Signal}

Up to now, the amplitude characteristic of the predicted spectrum has been discussed [21], but the phase characteristic has not been provided. In this section, the phase characteristic of the spectrum predicted by the band expansion method is discussed. The compressed pulse is calculated from the estimated received signal with an added $0.3 \mathrm{~ms}$ delay $\left(\theta_{\mathrm{T}}=0^{\circ}\right)$, and the band-expanded signal is derived from the compressed signal. Figure 9

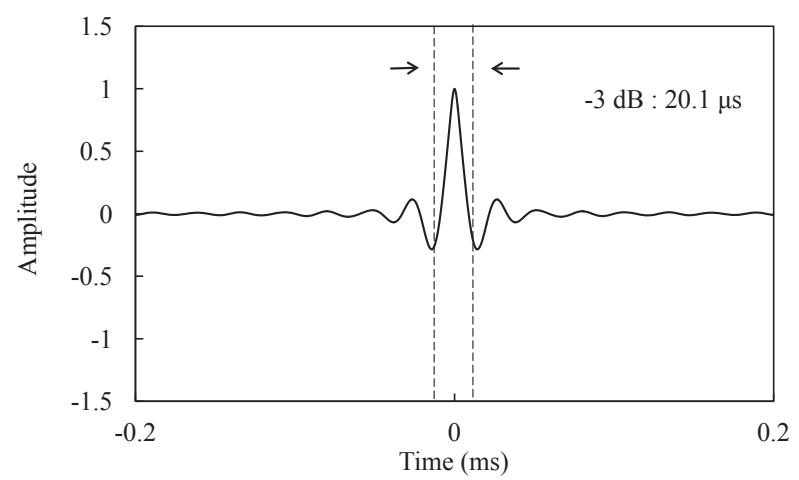

(a)

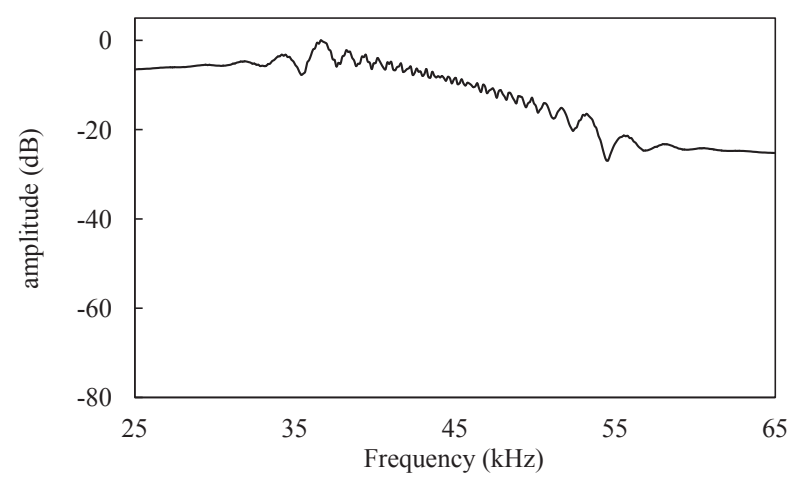

(b)

Fig. 8 Band-expanded signal derived from compressed signal $\left(\theta_{\mathrm{T}}=45^{\circ}\right)$ : (a) waveform and (b) spectrum.

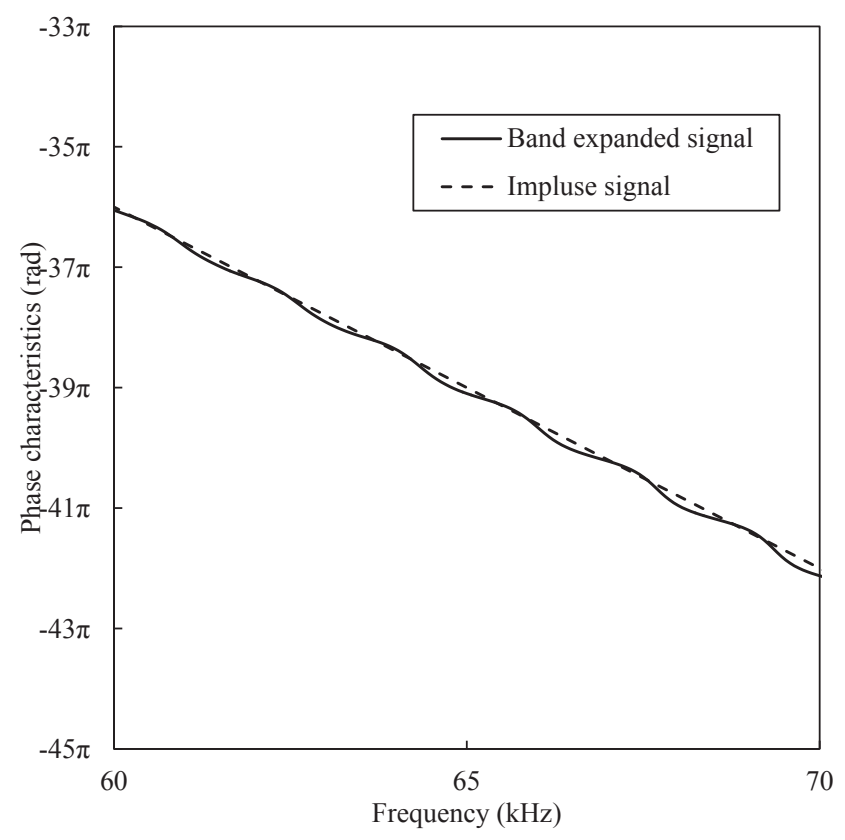

Fig. 9 Phase spectrum of band-expanded signal with added $0.3 \mathrm{~ms}$ time delay $\left(\theta_{\mathrm{T}}=0^{\circ}\right)$.

shows the phase spectrum of the band-expanded signal with an added $0.3 \mathrm{~ms}$ delay $\left(\theta_{\mathrm{T}}=0^{\circ}\right)$ and that of the impulse signal with a $0.3 \mathrm{~ms}$ delay. Here, the band width shown in Fig. 9 is the bandwidth with the lower SNR in the 


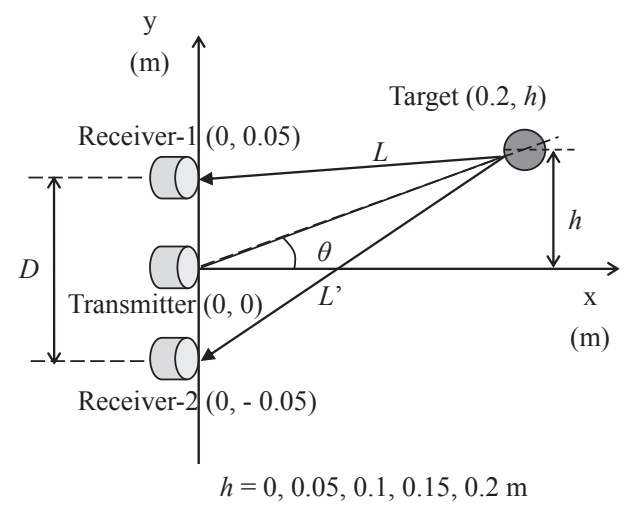

Fig. 10 Arrangement used for direction measurement.

compressed signal, and the result shown in the figure is the phase of the predicted spectrum calculated by the band expansion processing.

As shown in Fig. 9, the phase characteristics of the band-expanded signal are almost in agreement with those of the impulse signal. This result suggests that the phase spectrum is compensated by an amount corresponding to the time delay of the signal. Therefore, the compensation of predicted values for the spectrum with the lower SNR is efficient.

\section{EXPERIMENTAL CONDITION}

The arrangement used for direction measurement is shown in Fig. 10, and the procedure of direction measurement is shown in Fig. 11. The transmitter and the receivers are arranged parallel with a $50 \mathrm{~mm}$ interval, and the target is a cylinder with $50 \mathrm{~mm}$ diameter. We denote $L$ and $L^{\prime}$, as the propagation distances from the target to the two receivers and $D$ as the interval between the receivers. Here, If $D \ll L$, the angle $\theta$ can be approximately calculated as

$$
\theta=\sin ^{-1} \frac{\left|L-L^{\prime}\right|}{D}
$$

In the direction measurement, the efficiency of the band expansion method is discussed by considering the SNR and the evenness of the spectrum affected by the directivities of the transducers. Direction measurement using the compressed signal is compared with that using the bandexpanded signal. A transmitting signal with a peak-to-peak voltage of $2 \mathrm{~V}$ is employed. The angle is measured 20 times with the target placed at each location $(h=0,0.05$, $0.1,0.15,0.2 \mathrm{~m}$ ), and the errors of the deviation $E R R_{\mathrm{d}}$ are calculated as Eq. (12), where $N$ is the number of measurements, $\theta_{0}$ is the angle of the arrangement and $\theta_{m}$ is the angle calculated from the TOF.

$$
E R R_{\mathrm{r}}=\sqrt{\frac{\sum_{m=1}^{N}\left(\theta_{m}-\theta_{0}\right)^{2}}{N}}
$$

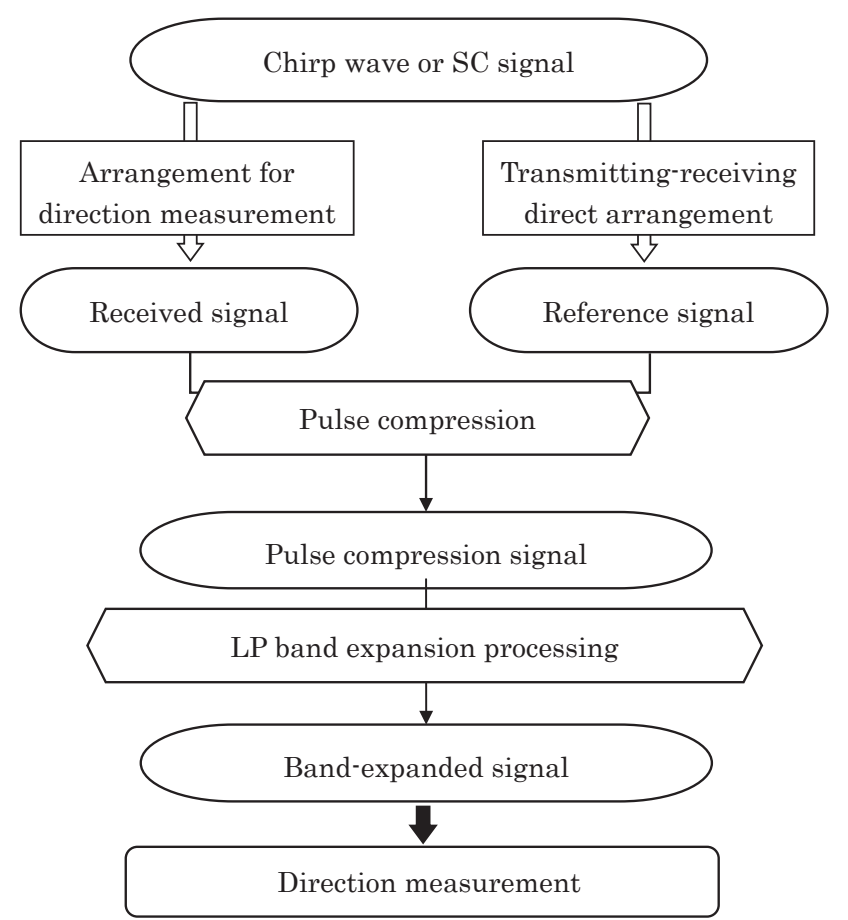

Fig. 11 Procedure of direction measurement.

\section{RESULTS OF DIRECTION MEASUREMENT AND DISCUSSION}

An example of a pulse compressed signal measured by Receiver- 2 when $\theta=0^{\circ}(h=0 \mathrm{~m})$ using the SC signal is shown in Fig. 12. The result shows that the $-3 \mathrm{~dB}$ pulse width of the SC signal is $53.3 \mu$ s. The band-expanded signal derived from Fig. 12 is shown in Fig. 13. As shown, the $-3 \mathrm{~dB}$ pulse width is $9.4 \mu \mathrm{s}$, and the pulse width is shortened to about $18 \%$ of that when using the SC signal. Because the spectrum is compensated by the predicted values calculated from the flat spectrum, the time resolution is improved. Here, $p=26$ is employed for direction measurement. Examples of a pulse compressed signal obtained using the SC signal when $\theta=45^{\circ}(h=0.2 \mathrm{~m})$ and the band-expanded signal are shown in Figs. 14 and 15, respectively. These results show that the $-3 \mathrm{~dB}$ pulse width of the pulse compressed using the SC signal and that of the band-expanded signal are $59.8 \mu$ s and $23.1 \mu \mathrm{s}$, respectively. In this arrangement, the pulse width is shortened to about 39\%; however, the efficiency of the band expansion is lower than that when the target is located at $\theta=0^{\circ}$. Because the received signal is affected by ultrasonic propagation characteristics including the directivity and the receiving SNR, the efficiency of the band expansion is decreased.

The accuracy of direction measurement is shown in Fig. 16. The accuracy of direction measurement using the SC signal is superior to that using the chirp wave. Furthermore, using the band-expanded signal, the accuracy 


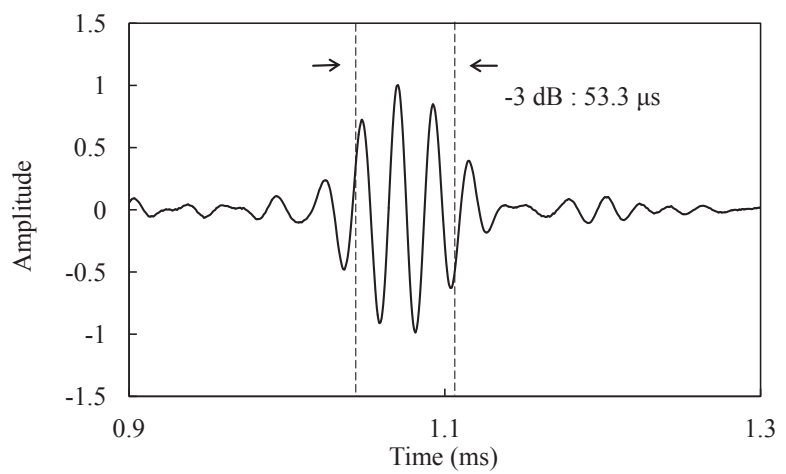

(a)

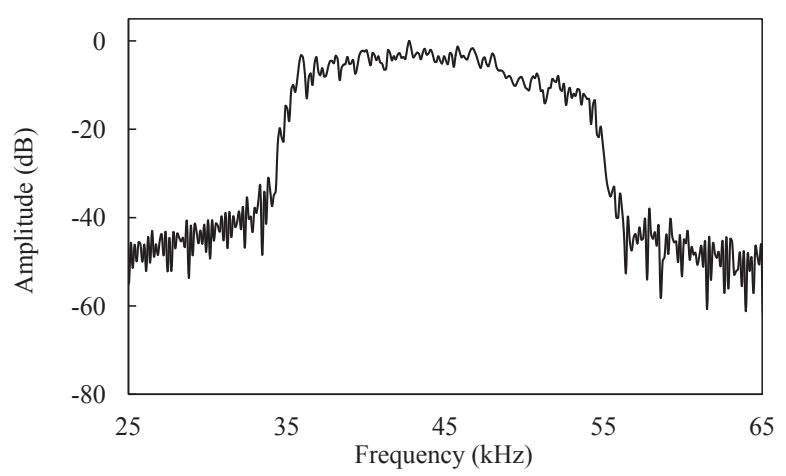

(b)

Fig. 12 Example of a compressed signal using the SC signal $\left(\theta=0^{\circ}\right)$ : (a) waveform and (b) spectrum.

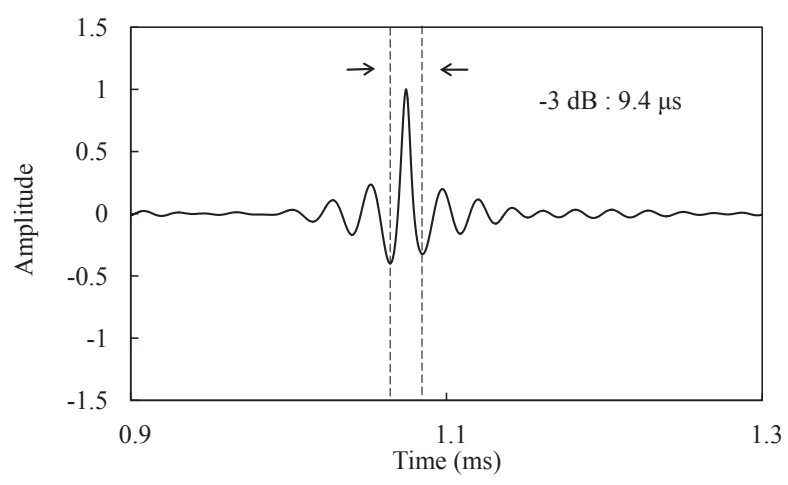

(a)

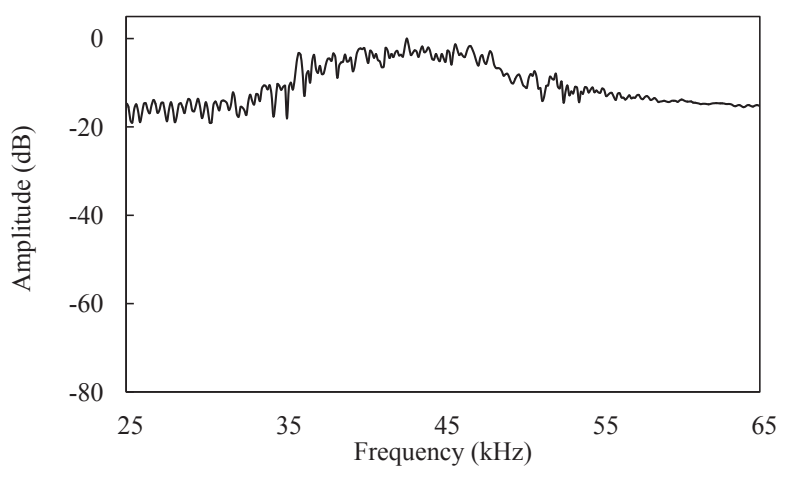

(b)

Fig. 13 Example of a band-expanded signal derived from the SC compressed signal $\left(\theta=0^{\circ}\right.$ ): (a) waveform and (b) spectrum.

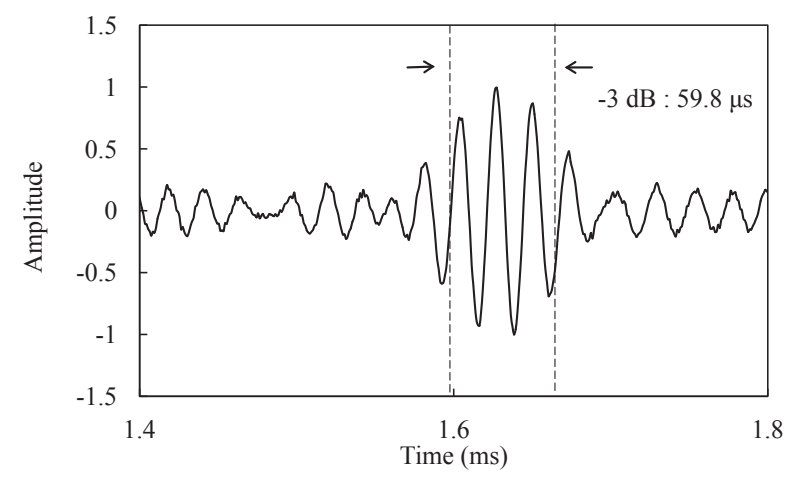

(a)

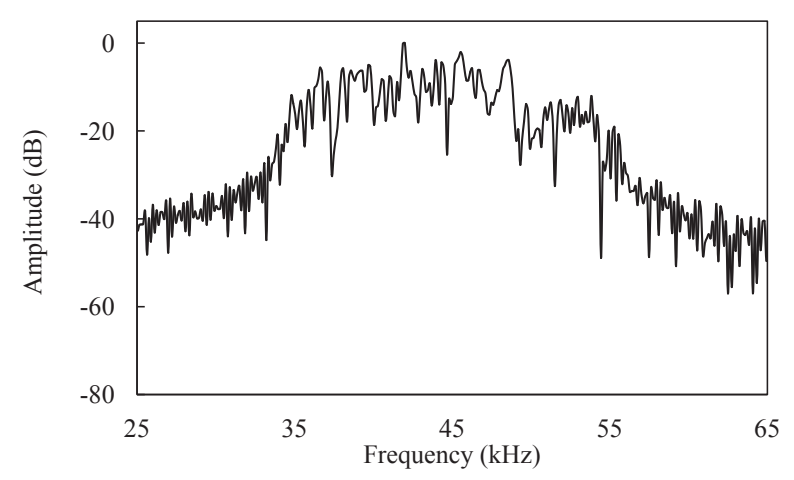

(b)

Fig. 14 Example of a compressed signal using the SC signal $\left(\theta=45^{\circ}\right)$ : (a) waveform and (b) spectrum.

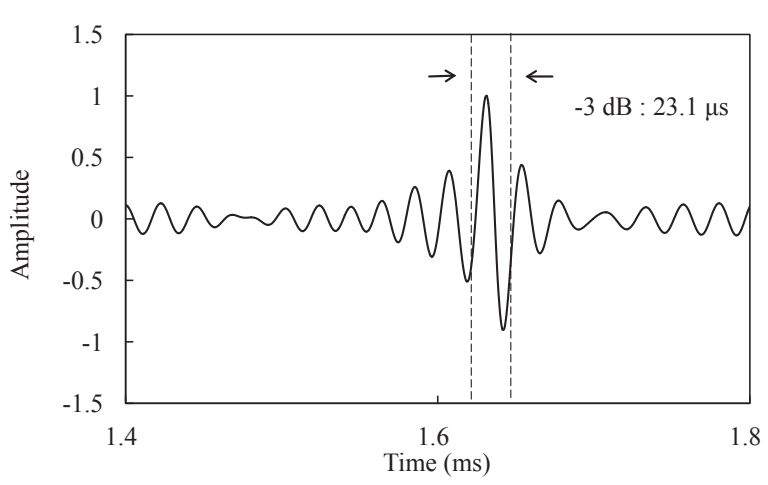

(a)

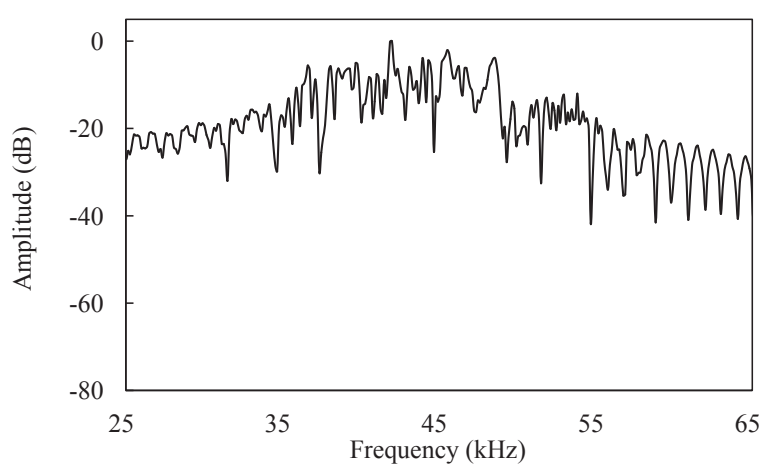

(b)

Fig. 15 Example of a band-expanded signal derived from the SC compressed signal $\left(\theta=45^{\circ}\right)$ : (a) waveform and (b) spectrum. 


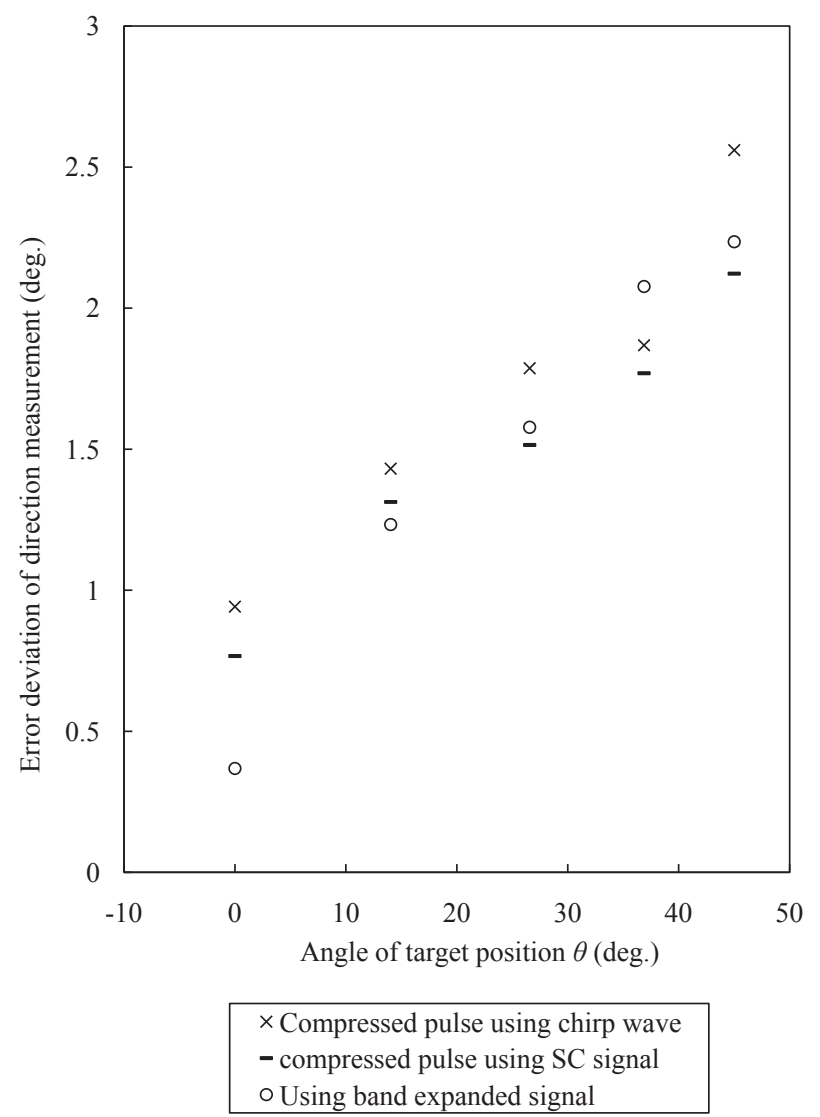

Fig. 16 Accuracy of direction measurement.

of direction measurement is superior to that using the SC signal when the target in the arrangement is located at $\theta=0$ and $14^{\circ}(h=0,0.05 \mathrm{~m})$. On the other hand, when the target is located at other values of $h$, the accuracy of direction measurement using the band-expanded signal is less than that using the SC signal. The accuracy of direction measurement using the band expansion method is improved when the target is located at a position where a signal with a flatter spectrum can be received; however, the accuracy of direction measurement is decreased when the target is located at a position where the spectrum of the received signal becomes uneven. These results suggest that the accuracy of TOF measurement is improved by using a sharp pulse derived from the LP band expansion processing with a flatter spectrum.

\section{CONCLUSIONS}

A band expansion method using a sensitivity-compensated (SC) signal and linear prediction (LP) processing has been proposed for higher-accuracy time-of-flight (TOF) measurements. In this paper, the efficiency of band expansion based on LP processing with an autoregressive (AR) model was discussed for two-dimensional direction measurement.

Accordingly, improvement of the time resolution was verified by simulation and experiment. In addition, the accuracies of direction measurement using the band expansion method were improved when a signal with a flatter spectrum could be received.

Future studies on the optimal parameters for the AR calculation, such as the LP order and the range of the spectrum used to calculate the LP coefficients, are required.

\section{ACKNOWLEDGMENT}

This work was partly supported by a MEXT Supported Program for the Strategic Research Foundation at Private Universities (S1311004).

\section{REFERENCES}

[1] J. L. Crowley, "World modeling and position estimation for a mobile robot using ultrasonic ranging," Proc. IEEE Int. Conf. Robotics and Automation, 2, 674-680 (1989).

[2] J. Borenstein and Y. Koren, "Noise rejection for ultrasonic sensors in mobile robot applications," Proc. IEEE Int. Conf. Robotics and Automation, 2, 1727-1732 (1992).

[3] S. Hirata, M. K. Kurosawa and T. Katagiri, "Accuracy and resolution of ultrasonic distance measurement with high-timeresolution cross-correlation function obtained by single-bit signal processing," Acoust. Sci. \& Tech., 30, 429-438 (2009).

[4] A. C. R. Alves and H. C. Junior, "Mobile ultrasonic sensing in mobile robot," IEEE 28th Annu. Conf. Ind. Electr. Soc., 4, 2599-2604 (2002).

[5] K. W. Jorg and M. Berg, "Mobile robot sonar sensing with pseudo-random codes," Proc. IEEE Int. Conf. Robotics and Automation, 4, 2807-2812 (1998).

[6] T. H. Gan, D. A. Hutchins, D. R. Billson and D. W. Schindel, "High-resolution, air-coupled ultrasonic imaging of thin materials," IEEE Trans. Ultrason. Ferroelectr. Freq. Control, 50, 1516-1524 (2003).

[7] T. H. Gan, D. A. Hutchins, R. J. Green, M. K. Andrews and P. D. Harris, "Noncontact, high-resolution ultrasonic imaging of wood samples using coded chirp waveforms," IEEE Trans. Ultrason. Ferroelectr. Freq. Control, 52, 280-288 (2005).

[8] P. Pallav, T. H. Gan and D. A. Hutchins, "Elliptical-Tukey chirp signal for high-resolution, air-coupled ultrasonic imaging," IEEE Trans. Ultrason. Ferroelectr. Freq. Control, 54, 1530-1540 (2007).

[9] T. Helleseth, "Crosscorrelation of m-sequences, exponential sums and Dickson polynomials," IEICE Trans. Fundam., E93A, 2212-2219 (2010).

[10] R. Toh and S. Motooka, "Target ranging using ultrasonic sensitivity-compensated signal and pulse compression," Jpn. J. Appl. Phys., 48, 07GB09 (2009).

[11] D. Chimura, R. Toh and S. Motooka, "Target ranging in water by using an FM-sensitivity-compensated signal and pulse compression," J. Marine Acoust. Soc. Jpn., 38, 61-71 (2011) (in Japanese).

[12] R. Toh, D. Chimura and S. Motooka, "Target ranging by using ultrasonic adaptive signal and pulse compression," Proc. Symp. Ultrason. Electron., 29, 213-214 (2008).

[13] D. Chimura, R. Toh and S. Motooka, "Expanding of the bandwidth of receiving signal by using adaptive transmitting signal," Proc. Autumn Meet. Acoust. Soc. Jpn., pp. 1457-1458 (2008) (in Japanese).

[14] D. Chimura, R. Toh and S. Motooka, "Accuracy of target ranging by using sensitivity compensated transmitting signal," Proc. Autumn Meet. Acoust. Soc. Jpn., pp. 1325-1326 (2009) (in Japanese). 
[15] R. Toh, D. Chimura and S. Motooka, "Speed measurement of moving object by using sensitivity compensated ultrasonic transmitting signal and pulse compression," Proc. Int. Congr. Acoust. (ICA 2010), 20, 23-27 (2010).

[16] D. Chimura, R. Toh and S. Motooka, "Speed measurement by using sensitivity compensated FM signal," Proc. Symp. Ultrason. Electron., 32, 73-74 (2011).

[17] R. Toh, D. Chimura and S. Motooka, "An experimental study on speed measurement using sensitivity compensated signal and linear prediction processing," Proc. IEEE Ultrason. Symp. Int., pp. 1461-1464 (2012).

[18] C. W. Harrison, "Experiments with linear prediction in television,” Bell Syst. Tech. J., 31, 764-783 (1952).

[19] J. Makhoul, "Spectral linear prediction: Properties and applications," IEEE Trans. Acoust. Speech Signal Process., 23, 283-296 (1975).
[20] R. Toh, D. Chimura and S. Motooka, "An experimental study on speed measurement using sensitivity compensated signal and linear prediction processing," Proc. IEEE Ultrason. Symp. Int., pp. 1461-1464 (2012).

[21] D. Chimura, R. Toh and S. Motooka, "Ultrasonic ranging in air using sensitivity-compensated signal with linear prediction," J. Acoust. Soc. Jpn. (J), 70, 7-15 (2014) (in Japanese).

[22] D. Chimura, R. Toh and S. Motooka, "Sensitivity compensated transmitting signal for direction measurement using pulse compression," Proc. Symp. Ultrason. Electron., 34, 121-122 (2013).

[23] D. Chimura, R. Toh and S. Motooka, "Ultrasonic direction measurement using sensitivity compensated transmitting signal and pulse compression," Proc. Int. Congr. Ultrason. (2015 $I C U)$, 70, 476-479 (2015). 